inhibitory substance in this case seems to be a large molecule, unlike the one which inhibits the growth of the Harding Passey melanoma in vitro (but not that of Chinese hamster fibroblasts). The molecular size of this last compound is less than 10,000 and is destroyed by trypsin or neuraminidase, and, according to $\mathrm{Dr}$ D. L. Dewey (Mount Vernon Hospital, Middlesex), it also causes a marked "maturation" of the melanoma cells in vitro.

The kinetics of the interlinked cell populations in haemopoietic tissue was discussed by Dr L. Lajtha (Holt Radium Institute, Manchester) who emphasized the techniques necessary for identification of specific chalone effects in this cell system. Finally, the possible mechanism of loss of specific tissue function control was discussed by Dr B. Rabin (University College, London) in an elegant demonstration of membrane changes produced by carcinogens, an illustration on how "chalone" control may be lost by the cells.

\section{CANCER RESEARCH Activating Carcinogens}

from our Cell Biology Correspondent ONE of the ironies of cancer research is that although chemical carcinogens, such as the polycyclic aromatic hydrocarbons, are chemically simpler structures than RNA or DNA tumour viruses, chemical carcinogenesis is less amenable to investigation than is viral carcinogenesis. Chemical carcinogenesis seems to be a hit and run affair, which does not depend on the persistence of the carcinogen once it has hit its target, whereas the maintenance of the transformation of cells infected with cancer viruses seems to depend on the continuous expression of viral "transforming" genes.

By exploiting strains of virus which carry conditional lethal mutations in the "transforming" genes and by identifying all the proteins specified by a tumour virus genome it should be possible to identify not only the transforming proteins but their cellular targets. The elucidation of the biochemistry of chemical carcinogens, by contrast, is a more formidable task. First, it must be determined whether a metabolite of any compound administered rather than the compound itself is the carcinogen and then its target must be identified. Added to which it is only comparatively recently that cultivated cells which reproducibly are susceptible to chemical carcinogenesis have become available.

Heidelberger and his colleagues, for example, have used cultivated mouse prostate cells with notable success to confirm Boyland's old suggestion that at least some polycyclic hydrocarbons are activated by being metabolized into $K$ epoxides. Their latest report shows that the $\mathrm{K}$ region epoxides of benzanthracene, dibenzanthracene and 3methylcholanthrene are both more cytotoxic and more carcinogenic for mouse prostate cells than are the parental hydrocarbons (Marquardt et al., Cancer Res., 32, 716; 1972). The $\mathbf{K}$ region epoxides of methylbenzanthracene and the bromomethyl substituent by contrast are less potent carcinogens than are the parental molecules.

In a subsequent report (ibid., 721), Marquardt and Heidelberger describe experiments in which mouse prostate cells were cultivated with lethally irradiated feeder layers of either rat or mouse embryo cells while exposed to various polycyclic hydrocarbons. The idea behind these experiments was to test the effect on cytotoxicity and carcinogenicity of the hydrocarbons for prostate cells of feeder cells either more or less able to metabolize the hydrocarbons than the prostate cells.

As expected, the addition of feeder layers of cells which readily metabolize the hydrocarbons to $\mathrm{K}$ epoxides resulted in a significant increase in the cytotoxicity and carcinogenic potential of those hydrocarbons which need to be metabolized to an active epoxide. Feeder layers of human skin cells, which metabolize these compounds to a lesser extent than do the mouse prostate cells, also failed to alter the yield of transformed mouse cells. Moreover, the addition of drugs which either induce increased or decreased intracellular levels of mixed-function oxidase activities had the expected effect on the yield of transformants obtained with given doses of methylcholanthrene and benzanthracene. These data therefore also support the hypothesis that at least some non-methylated, polycyclic, aromatic hydrocarbons have to be metabolized to $\mathbf{K}$ epoxides in order to be carcinogenic, and of course the feeder layer technique provides an assay for the extent to which cells from various sources can bring about this step.

Asbestos, like hydrocarbons, is a carcinogen and considerable effort has gone into trying to determine whether or not asbestos per se or some contaminant is responsible for neoplastic transformation. According to Starton and Wrench, who report the results of experiments with rats started more than 2 years ago (J. Nat. Cancer Inst., 48, $979 ;$ 1972), the carcinogenicity of asbestos may depend on its physical structure rather than its chemistry.

Starton and Wrench compared the carcinogenicity of crocidolite, amosite and chrysotile asbestoses milled to various extents and administered on a fibreglass vehicle. They believe that under their conditions "the structural integrity of the asbestos fiber above that of the finest submicroscopic fibril is essential to carcinogenicity and that neither contaminants nor chemical components are likely factors". If this is the case it will be interesting to see if similarly sized fibres of other materials of comparable durability are carcinogenic.

\title{
Granulocyte and Macrophage Differentiation
}

Elucidating the molecular basis of cell differentiation is currently one of the chief preoccupations of many biologists and of the several systems that have attracted attention perhaps the most amenable to further detailed investigation is the differentiation in vitro of haematopoietic stem cells into mature granulocytes and macrophages. Sachs and his colleagues are among the pioneers in this field and their latest contribution appears in Nature New Biology next week (June 28).

Sachs and his colleagues have previously shown that a protein factor with a molecular weight of between 65,000 and 70,000 which is released by cultivated mouse fibroblasts will, when supplemented with an adenine nucleotide cofactor, induce normal mouse stem cells to differentiate into granulocytes and macrophages. Sachs et al. have now investigated the ability of this factor to induce the differentiation in vitro of myeloid leukaemic stem cells from mice. Crude preparations of the inducing protein-serum-free medium conditioned by mouse fibroblastsincrease some seven to eight-fold the cloning efficiency in soft agar of the myeloid stem cells; crude factor also causes about 5 per cent of the colonies to differentiate to granulocytes and 45 per cent to differentiate to macrophages.

By ultrafiltration Sachs et al. then separated the protein factor in the crude preparations from the low molecular weight nucleotide cofactor and assayed the ability of the protein inducer, in the absence of cofactor, to stimulate differentiation of normal and leukaemic stem cells. They found that whereas the induction of differentiation of normal stem cells depends on cofactor as well as inducer, colonies of myeloid leukaemic stem cells differentiate when exposed to the protein inducer alone, and addition of adenine nucleotides failed to increase the proportion of differentiated colonies.

It seems therefore that unlike normal stem cells myeloid leukaemic stem cells of mice produce the adenine cofactor required to potentiate the differentiation-inducing protein. At the very least this finding may form the basis of a diagnostic test for myeloid leukaemia blast cells. 